\title{
An Extraordinary Presentation of a Human Psittacosis: Mimicing Acute Monocytic Leukemia
}

\author{
Fatos Atilla ${ }^{1}$, Melike Ordu² , and Burcu Baran Ketencioglu ${ }^{2}$ \\ ${ }^{1}$ Affiliation not available \\ ${ }^{2}$ Aksaray Universitesi
}

May 5, 2020

\begin{abstract}
Chlamydia psittaci is an intracellular organism causing psittacosis which is found in psittacine birds and can be transmitted to humans by inhalation or direct contact. Here we present a case of human psittacosis mimicing acute monocytic leukemia which demonstrates an extraordinary clinical presentation of psittacosis.
\end{abstract}

Key Clinical Message: Human psittacosis should be consider in cases who show typical and atypical respiratory signs hence pigeon keeping or pigeon fancying as a hobby is common in Turkey. Detailed medical history should be taken in suspicious cases.

\section{Introduction}

Chlamydia psittaci is an obligate intracellular bacterium which causes psittacosis in psittacine birds and can be transmitted to humans by inhalation or direct contact. Birds are the primary reservoir, but transmission from other animals has also been reported. Virtually all pet birds can carry C. psittaci, but psittacine birds are the most likely to be infected. There are 10 known genotypes based on sequencing of the major outer protein gene and each genotype has host preferences and virulence characteristics ${ }^{1}$. Psittacosis which was estimated to cause approximately 1 percent of cases of community-acquired pneumonia has been recognized throughout the world ${ }^{2}$. The precise incidence and prevalence of psittacosis is difficult to establish, likely due to lack of reachable sensitive and specific diagnostic tests ${ }^{3}$. Psittacosis has been described in all age groups but most commonly seen in young and middle-aged adults. A marked predilection for males is noted, presumably related to increased potential for exposure ${ }^{4}$. Local outbreaks which related to pet shops, aviaries and veterinary facilities have been described ${ }^{5,6}$.

Patients usually present with respiratory symptoms likely dry cough due to pulmonary infection with varying severity. The most frequently reported clinical symptoms are high fever, chills, malaise, headache, myalgia, non-productive cough and respiratory distress ${ }^{7}$. Infection can also be asymptomatic and other less common but important symptoms include pharyngitis, diarrhea, and altered mental status can be seen. Patients usually have a history of bird exposure. The incubation period is usually between 5 and 14 days but can be as long as 39 days $^{8}$. Unusual presentations which complicates making the precise diagnosis may rarely be seen. Variable clinical symptoms may arise with involvement in a number of different organs. Cold agglutinins, hemolytic anemia, acute thrombocytopenic purpura, severe pancytopenia secondary to hemophagocytic syndrome and thrombotic thrombocytopenic purpura have been described as hematologic complications $^{9,10}$. But these are uncommon manifestations. 
Laboratory studies may reveal leukocytosis with toxic granulation or a left shift. The erythrocyte sedimentation rate (ESR) and C-reactive protein (CRP) are very often elevated. The liver enzymes are abnormal in approximately half of hospitalized patients. Mild elevation of serum creatinine and blood urea nitrogen and hyponatremia is quite common. The chest radiograph is usually abnormal most often with lobar changes ${ }^{11}$. Pleural effusions are rare but can occur. On high-resolution computed tomography, the pulmonary infiltrates may be nodular surrounded by ground-glass opacities ${ }^{12}$. Serology is the principal method of confirming the diagnosis. Microimmunofluorescent antibody test (MIF) is the most sensitive and specific serologic test for C. psittaci but is only available in specialty laboratories ${ }^{13}$. A fourfold rise in antibodies or an immunoglobulin (Ig)M antibody titer [?]16 is interpreted as diagnostic, but antibody titer of [?]32 is preferable for a sufficient diagnosis. However, negative serology in hospitalized psittacosis patients is not uncommon. The current serological tests are less sensitive than polymerase chain reaction (PCR). PCR methods give a rapid and specific diagnosis. It is not only more sensitive than culture and serology, it also allows genotyping. Unfortunately PCR is not available in many healt care institutions in Turkey.

The tetracyclines including doxycycline or tetracycline hydrochloride are preferred for the treatment of psittacosis. This treatment must be continued during a period of at least 10-14 days in order to be effective and to prevent relapse. Other agents like macrolides and quinolones may also have been proposed as alternatives but they are less active against C. psittaci than tetracyclines and macrolides ${ }^{14}$.

\section{Case Report}

A 70-year old male was admitted to the hospital with the symptoms of fatigue, myalgia, fever up to $39^{\circ} \mathrm{C}$ and non-productive cough since more than ten days. He had been treated with oral amoxicillin/clavulanate of $1000 \mathrm{mg}$ BID for seven days. But the patient stated that symptoms were worsened under the treatment. His medical history included chronic obstructive pulmonary disease (COPD) with 40 year of heavy smoking which was reduced for the last few years. There were no known allergic diseases.

At the time of admission his vital signs of blood pressure, respiration and heart rates were stable hence he was hospitalized at the internal medicine ward. Axillar temperature was $38.6^{\circ} \mathrm{C}$ and blood oxygen level was $86 \%$ on pulse oximetry. Physical examination revealed petechiaes on bilateral forelegs. Auscultation of the lungs showed bilateral coarse rales/crackles. On some areas of lungs bronchial breath sounds were also audible. Rest of systemic clinical examination showed no abnormalities.

Blood analysis showed leucocytosis $(12400 / \mu \mathrm{L})$ with relative monocytosis $(2330 / \mu \mathrm{L})$, mild anemia $(9.5 \mathrm{gr} / \mathrm{dL})$ and severe thrombocytopenia $(13000 / \mu \mathrm{L})$. Peripheral blood smear showed numerous promonocytes and monocytes that have folded and convoluted nuclei and a finely granulated cytoplasm without vacuoles (figure 1). Coagulation tests were in normal range. There was an elevated C-reactive protein (CRP) (126 mg/L) and a sedimentation rate of $84 \mathrm{~mm} / \mathrm{h}$. Liver enzymes were slightly elevated, with an SGOT of $57 \mathrm{U} / \mathrm{L}$, an SGPT of $66 \mathrm{U} / \mathrm{L}$, a GGT of $104 \mathrm{U} / \mathrm{L}$ and alkaline phosphatase of $365 \mathrm{U} / \mathrm{L}$. Bilirubin levels were normal. Auto-immune serology including rheumatoid factor, anti-Cyclic Citrullinated Peptide antibodies and Anti-Nuclear Antibodies were all negative. Viral and bacterial serology were negative. A chest radiograph revealed extensive pleuropneumonia in bilateral lungs and chest CT showed multiple peripheral ground glass opacities with consolidation in both lungs (figure 2). Bone marrow biopsy was performed given acute monocytic leukemia was the potential initial diagnosis. Normocellular bone marrow with relative monocytosis, increased histiocytes with hemophagocytosis was revealed. Immunohistochemical staining showed normal promonocytes and monocytes without monoblasts (figure 3). Acute leukemia which was considered as an initial diagnosis was excluded. Pulmonary function tests showed moderate COPD with FEV1/FVC (Tiffeneau Index) of 60 and a reduced Forced Vital Capacity (FVC). Transthoracic cardiac echography revealed no abnormalities. Urine culture, sputum culture and repeated blood cultures revealed no specific pathogen.

The patient had been keeping approximately a hundred pigeons as a pet for about five years. In the past birds had some illnesses which were treated by the veterinary physician. But there was no demonstrated documents about diagnoses and treatments. With the given clinical evidences psittacosis was considered. An antibody detection of C. psittaci assay was performed at the private laboratory without under social 
reimbursement. Psittacosis was subsequently confirmed by specific IgG immunofluorescence testing. The serum had an IgM titre of $1: 16$, and elevated titres of $\operatorname{IgG}(1: 512)$ to C.psittaci, indicating an acute infection. Genotype-specific real-time PCR could not be performed due to unavailability. After one-week course of levofloxacin, as clinical improvement was lacking doxycycline at a dose of 100mg BID was initiated. Cytopenias due to hemophagocytosis was gradually resolved. Doxycycline was continued for three-week course in order to prevent relapse. Clinical and laboratory response was achieved. Blood values were all became normal ranges with being resolved of monocytosis. The patient fully recovered and discharged.

\section{Conclusion}

Here we present a case of human psittacosis mimicing acute monocytic leukemia which demonstrates an extraordinary clinical presentation of psittacosis. Some hematologic complications of disease have been described: Cold agglutinins, hemolytic anemia, acute thrombocytopenic purpura, severe pancytopenia secondary to hemophagocytic syndrome and thrombotic thrombocytopenic purpura. Hematologic complications usually occur in patients with fulminant disease. Disseminated intravascular coagulation has been described by two different authors as one of the lethal hematologic complication ${ }^{15,16}$. According to published paper the case was died due to massive thrombosis and respiratory failure and psittacosis was subsequently confirmed by complement fixation tests ${ }^{16}$. Other case with disseminated intravascular coagulation was fully recovered after initiation of heparin and doxycycline ${ }^{15}$. Severe pancytopenia secondary to hemophagocytic syndrome similarly to our case has also been described ${ }^{9}$. Cold agglutinins are sometimes noted in psittacosis, but hemolytic anemia with jaundice is rare. A case presenting with jaundice and the clinical symptoms of an atypical pneumonia which was attributed to Chlamydia psittaci with serologic results has described. Under the literature review on psittacosis and haematological complications, authors concluded that cold agglutinins may be found in association with psittacosis, but a concomitant haemolytic anaemia is very rare ${ }^{10}$. Hematologic complications of psittacosis occurs quite rare and are defined based on case reports. According to our review of literature monocytosis demonstrating like acute monocytic leukemia has not been described. The patient was fully recovered after three-week course of doxycycline.

Human psittacosis should be consider in cases who show typical and atypical respiratory signs. In recent years pigeon keeping or pigeon fancying as a hobby is common in Turkey. Therefore detailed medical history including occupational exposure to psittacine birds should be taken in all cases even with unusual clinical presentation. Systematic review of clinical and laboratory signs are fundamental to reach the precise diagnosis. Antibody detection assays are the only diagnostic tools in many institutions to define psittacosis in humans. But in Turkey antibody detection assays are unavailable in most of the public hospitals. PCRbased testing which is highly sensitive and spesific is very expensive and very hard-to-reach. In our case antibody detection assay could not be perfomed in our institution. The blood samples were sent to private laboratory which does not have an aggrement with social reimbursement. Unfortunately these difficulties during diagnostic process make the psittocosis infection being reported vastly underestimated. The real burden of this disease remain undetected.

\section{Funding sources}

This research did not receive any specific grant from funding sources in the public, commercial, or not-forprofit sectors.

\section{Author Contribution Statement}

FDA wrote and review the manuscript, MO editted the figures and review the manuscript, BBK review the manuscript and added contribution in evaluation of CT images.

\section{References}

1 Wolff, B. J. et al. Chlamydia psittaci comparative genomics reveals intraspecies variations in the putative outer membrane and type III secretion system genes. Microbiology 161, 1378-1391, doi:10.1099/mic.0.000097 (2015). 
2 Hogerwerf, L., B, D. E. G., Baan, B. \& W, V. D. H. Chlamydia psittaci (psittacosis) as a cause of community-acquired pneumonia: a systematic review and meta-analysis. Epidemiol Infect 145 , 3096-3105, doi:10.1017/S0950268817002060 (2017).

3 Rane, V. et al. Underdiagnosis of Chlamydia trachomatis and Chlamydia psittaci revealed by introduction of respiratory multiplex PCR assay with Chlamydiaceae family primers. Diagn Microbiol Infect Dis 90 , 163-166, doi:10.1016/j.diagmicrobio.2017.11.013 (2018).

4 Yung, A. P. \& Grayson, M. L. Psittacosis-a review of 135 cases. Med J Aust 148, 228-233 (1988).

5 Gaede, W. et al. Chlamydophila psittaci infections in humans during an outbreak of psittacosis from poultry in Germany.Zoonoses Public Health 55 , 184-188, doi:10.1111/j.1863-2378.2008.01108.x (2008).

6 Hedberg, K. et al. An outbreak of psittacosis in Minnesota turkey industry workers: implications for modes of transmission and control. Am J Epidemiol 130, 569-577 (1989).

7 Beeckman, D. S. \& Vanrompay, D. C. Zoonotic Chlamydophila psittaci infections from a clinical perspective. Clin Microbiol Infect15, 11-17, doi:10.1111/j.1469-0691.2008.02669.x (2009).

8 Grayston, J. T. \& Thom, D. H. The chlamydial pneumonias. Curr Clin Top Infect Dis 11, 1-18 (1991).

9 Wong, K. F., Chan, J. K., Chan, C. H. \& Lim, W. W. Psittacosis-associated hemophagocytic syndrome. Am J Med91 , 204-205 (1991).

10 Timmerman, R. \& Bieger, R. Haemolytic anaemia due to cold agglutinins caused by psittacosis. Neth J Med 34, 306-309 (1989).

11 Kuwabara, M. et al. [Clinical features of 36 cases of psittacosis]. Kansenshogaku Zasshi 64, 498-503 (1990).

12 Hochhegger, B. et al. Psittacosis presenting as a halo sign on high-resolution computed tomography. $J$ Thorac Imaging 24 , 136-137, doi:10.1097/RTI.0b013e318191987e (2009).

13 Wreghitt, T. Chlamydial infection of the respiratory tract.Commun Dis Rep CDR Rev 3, R119-124 (1993).

14 Mandell, L. A. et al. Infectious Diseases Society of America/American Thoracic Society consensus guidelines on the management of community-acquired pneumonia in adults. Clin Infect Dis44 Suppl 2, S27-72, doi:10.1086/511159 (2007).

15 Hamilton, D. V. Psittacosis and disseminated intravascular coagulation. Br Med J 2, 370 (1975).

16 Laidlaw, E. \& Mulligan, R. A. Letter: Psittacosis and disseminated intravascular coagulation. $\mathrm{Br} \mathrm{Med} J$ 2, 688 (1975). 


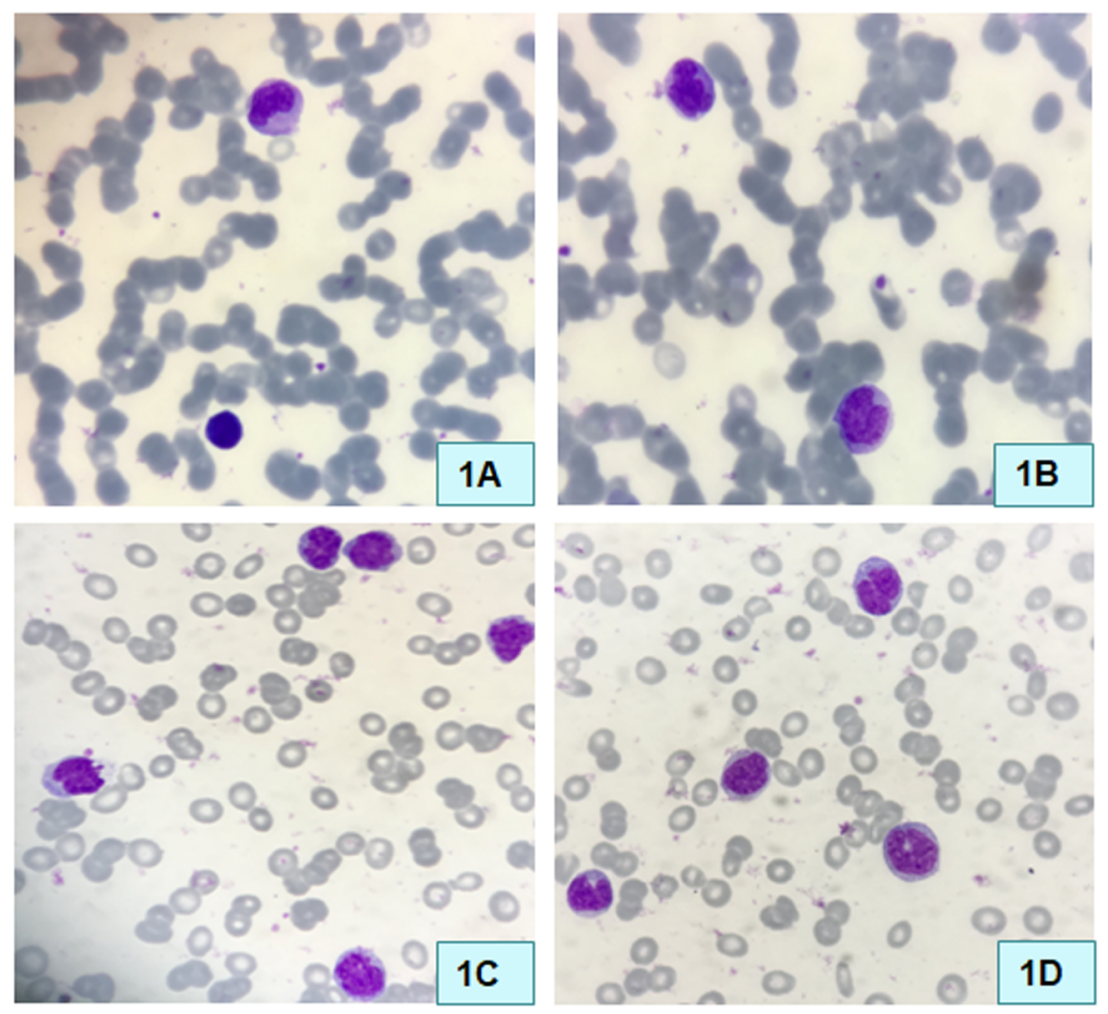

Figure 1: Four of all seperated figures of peripheral blood smear shows numerous promonocytes and monocytes that have folded and convoluted nuclei and a finely granulated cytoplasm without remarkable vacuoles.

IA and IB: Thick area of blood smear. IC and IC: OptimaVmedial area of blood smear.

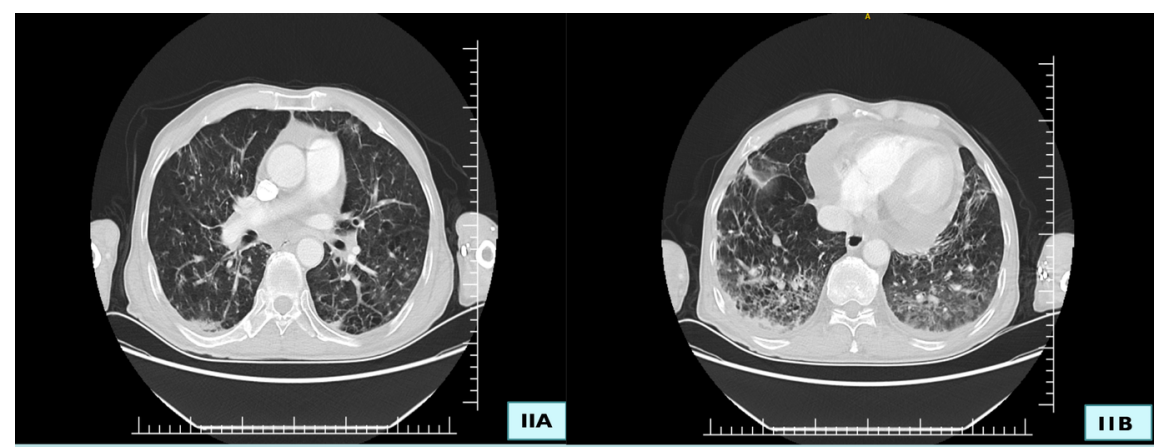

Figure IIA: Bilateral emphysema, bronchial dilatation, and chronic fibrotic changes are present in both lungs. Multifocal ground glass density nodules are observed.

Figure IIB: In the lower lobes, there is significant increase in the presence of ground glass opacities, consolidation and reticular density. Minimal degree of pleural and pericardial effusion is observed. 


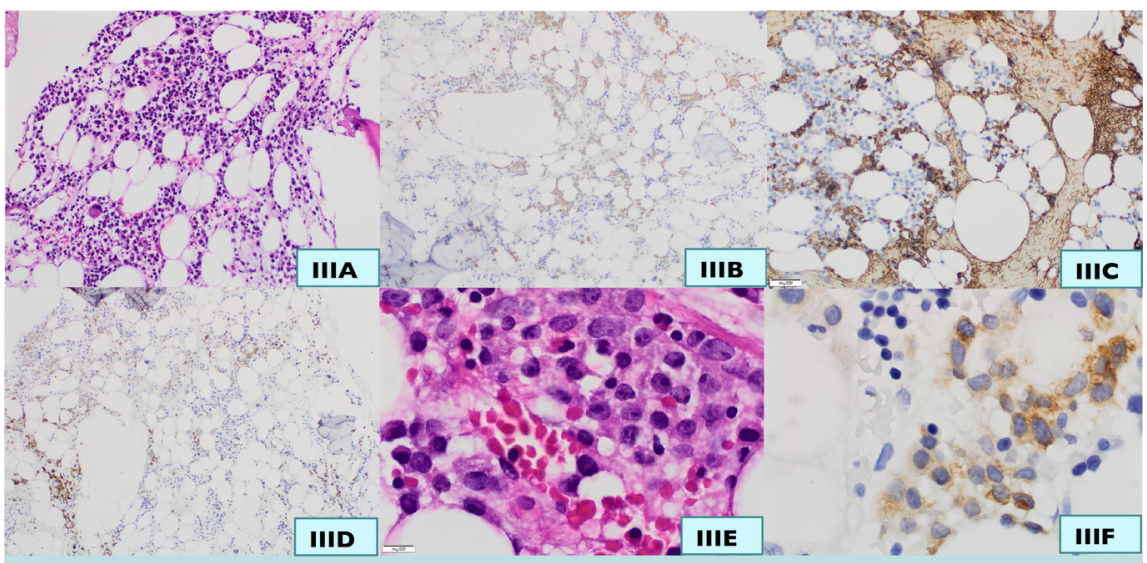

Figure III: Six of all bone marrow biopsy sections revealed normocellularity with normal erythroid, myeloid and megakoryocyte series. On paratrabecular and intertrabecular spaces numerous promonocytes and monocytes were seen without abnormal immunohistochemical stanining.

IIIA: Hematoxylen Eozin Stain (20x10) IIIB: Myeloperoxidase stain (20x10) IIIC: Glycoforin stain (20x10) IIID: Lysozyme(20x10) IIIE: Hematoxylen Eozin Stain (100x10) IIIF: Lysozyme (100x10) 